\title{
Geared Topological Metamaterials with Tunable Mechanical Stability
}

\begin{abstract}
Anne S. Meeussen, Jayson Paulose, and Vincenzo Vitelli*
Instituut-Lorentz, Universiteit Leiden, 2300 RA Leiden, The Netherlands

(Received 17 March 2016; revised manuscript received 21 July 2016; published 8 November 2016)

The classification of materials into insulators and conductors has been shaken up by the discovery of topological insulators that conduct robustly at the edge but not in the bulk. In mechanics, designating a material as insulating or conducting amounts to asking if it is rigid or floppy. Although mechanical structures that display topological floppy modes have been proposed, they are all vulnerable to global collapse. Here, we design and build mechanical metamaterials that are stable and yet capable of harboring protected edge and bulk modes, analogous to those in electronic topological insulators and Weyl semimetals. To do so, we exploit gear assemblies that, unlike point masses connected by springs, incorporate both translational and rotational degrees of freedom. Global structural stability is achieved by eliminating geometrical frustration of collective gear rotations extending through the assembly. The topological robustness of the mechanical modes makes them appealing across scales from engineered macrostructures to networks of toothed microrotors of potential use in micromachines.
\end{abstract}

DOI: 10.1103/PhysRevX.6.041029

Subject Areas: Mechanics, Metamaterials, Soft Matter

A machine channels power into specific, desired motions. This task is accomplished through mechanisms: rigid parts whose degrees of freedom are constrained by their assembly so that only the desired motion is allowed. Gears or cogwheels have been fundamental components of mechanisms since ancient times, underpinning the earliest known machines [1] and driving the engines of the Industrial Revolution [2]. Moreover, the microfabrication and propulsion of toothed rotors is a key challenge along the path to feasible nanomachines [3-7].

Mechanisms often underlie the behavior of mechanical metamaterials-artificial structures whose mechanical properties arise from the geometry and arrangement of their building blocks [8,9]. An appropriately designed mechanism or soft motion of the repeating unit cell can translate into an unusual bulk property such as a negative Poisson ratio [10,11], a vanishingly small shear modulus $[12,13]$, a tunable vibrational response [14], or highgradient elasticity [15]. More generally, the design of a mechanical metamaterial consists of constraining the available degrees of freedom so that a desired performance is achieved, much like a simple machine. The degrees of freedom and associated constraints may arise from simple springs or rigid beams connected by free hinges, or from more complicated elements such as flexible beams and blocks of various shapes. Despite the strong parallels between machines and metamaterials, however, the

\footnotetext{
*vitelli@lorentz.leidenuniv.nl
}

Published by the American Physical Society under the terms of the Creative Commons Attribution 3.0 License. Further distribution of this work must maintain attribution to the author(s) and the published article's title, journal citation, and DOI. potential for gears as building blocks of metamaterials and complex mechanisms remains unexplored.

Here, we demonstrate metamaterials that use periodic arrangements of gears and links to independently tune local flexibility and global stability. These assemblies of rigid elements harbor zero-energy mechanical modes (free motions as well as stress-bearing states) that owe their existence to topological invariants characterizing the mechanical excitations of the periodic structure. First introduced in isostatic spring networks [16-18], topological mechanical modes are insensitive to a wide range of structural perturbations like their counterparts in electronics $[19,20]$, photonics [21], and acoustics [22-29]. This feature makes them desirable for building metamaterials with robust mechanical motions and stress-bearing states localized to edges, defects, and domain walls [30-34]. While the topology of the excitation spectrum allows us to sculpt spatially nonuniform mechanical modes, the real-space topology of the link network and the rotational degrees of freedom are exploited to eliminate uniform deformations known as Guest-Hutchinson modes $[35,36]$ that generically lead to a lack of elastic stability in topological mechanical structures based on spring networks [37].

Geared Maxwell networks. -The fundamental building block of our designs is a pair of gears mounted on a solid link, as shown in Fig. 1(a). Each gear has three independent degrees of freedom: displacements along the two planar directions and a rotation. Each link constrains the distance between the connected gears, and also prevents motions that involve the gears sliding against each other [Fig. 1(b)]. The metamaterials we envision have a carefully chosen periodic structure, realized in assemblies of metal links with axles coupled by gears mounted on them [Fig. 1(c)]. Links of varied lengths are obtained from gears of a single 


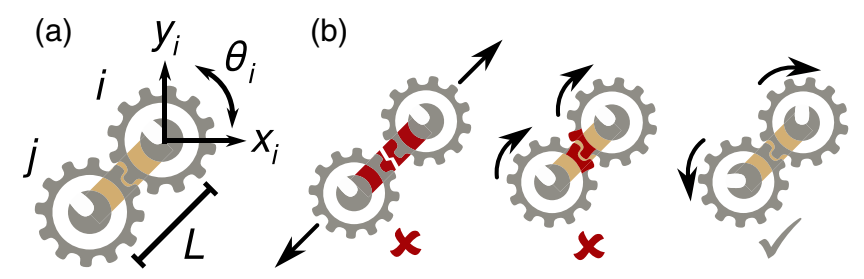

(c)

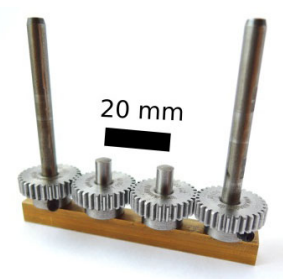

(d)

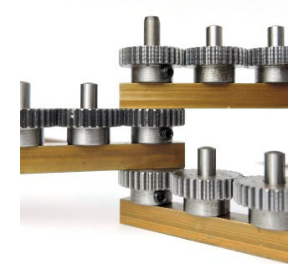

(e)

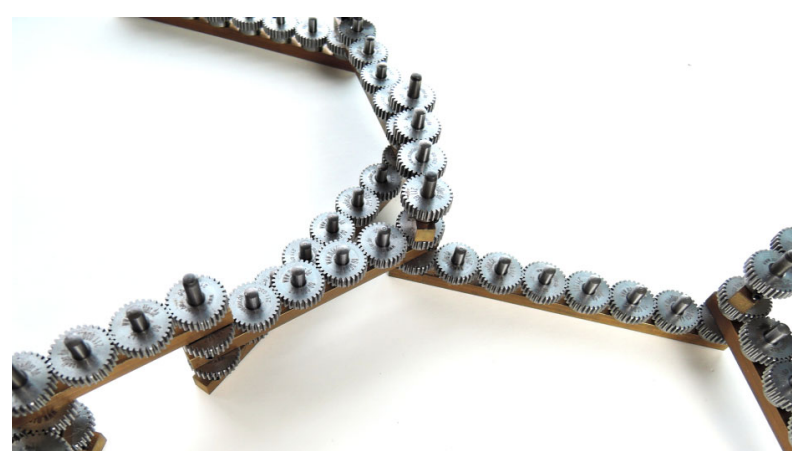

FIG. 1. Degrees of freedom and constraints in geared metamaterials. (a) The materials are built up of nodes with 3 degrees of freedom each: two translational $\left(x_{i}, y_{i}\right)$ and one rotational $\left(\theta_{i}\right)$. Nodes $i$ and $j$ are coupled by a single link, represented by the gray bar and the pair of gears that rotate together with the nodes. (b) The link constrains the distance between the nodes, and prevents rotations of the nodes in the same direction. However, counterrotations are allowed. (c) Realization of such an element using metal parts. The degrees of freedom associated with the long axles are coupled by gears mounted on a solid bar. The two gears at the ends rotate together with the long axles. The shear constraints are enforced using an even number of small gears to avoid self-intersections. (d) A joint that shares a single set of degrees of freedom, associated with the long axle, among three links. The links are stacked vertically to avoid self-intersections. (e) A unit cell consisting of a specific arrangement of six links that, when repeated, would form a geared metamaterial.

size by chaining even numbers of gears together. The relevant degrees of freedom, associated with the long axles at the ends, are shared among bars at junction nodes [Fig. 1(d)].

To describe the zero-energy modes of such a gear network, we first relax the condition of perfect rigidity and consider links with finite extensibility in the longitudinal and shear directions. Given displacements $u_{p, i}$ along the degree of freedom $p \in\{x, y, \theta\}$ of node $i$ (out of $N$ nodes), we define the longitudinal extension $e_{l, m}$ as the change in length of link $m$ (out of $N_{b}$ links) and the shear extension $e_{s, m}$ as the transverse deformation of the link ends that is uncompensated by gear counterrotation.
The compatibility matrix $\mathbf{C}$, described in detail in Appendix A, relates the $2 N_{b}$-dimensional vector of extensions $\mathbf{e}=\left(e_{l, 1}, e_{s, 1}, e_{l, 2}, \ldots\right)$ to the $3 N$-dimensional displacement vector $\mathbf{u}=\left(u_{x, 1}, u_{y, 1}, u_{\theta, 1}, u_{x, 2}, \ldots\right)$ via $\mathbf{C u}=\mathbf{e}$. Its transpose, the equilibrium matrix, relates forces and torques acting on the nodes $\mathbf{f}=\left(f_{x, 1}, f_{y, 1}, \tau_{1}, f_{x, 2}, \ldots\right)$ to a generalized stress vector $\mathbf{t}=\left(t_{1}, s_{1}, t_{2}, \ldots\right)$ of link tensions $t_{m}$ and shear forces $s_{m}$ via $\mathbf{C}^{\mathbf{T}} \mathbf{t}=\mathbf{f}$. Zero modes of the structure are displacements that lie in the null space of $\mathbf{C}$; in a structure where link extension and shearing are rigidly constrained, these are the only allowed motions. Members of the null space of $\mathbf{C}^{\mathbf{T}}$ are called states of self-stress, combinations of tensions and shears that leave all nodes in equilibrium. The number of zero modes $n_{m}$ is related to the number of states of self-stress $n_{\mathrm{ss}}$, via the MaxwellCalladine index theorem [16,38]:

$$
n_{m}-n_{\mathrm{ss}}=3 N-2 N_{b} .
$$

Our approach relies on the unique properties of isostatic structures, for which degrees of freedom and constraints are perfectly balanced-the so-called Maxwell criterion for structural stability [39]. In gear networks, this happens when $3 N=2 N_{b}$ or, equivalently, when the network has average coordination $z=2 N_{b} / N=3$. Equation (1) highlights the special status of such Maxwell lattices [37]: zero modes must be accompanied by an equal number of states of self-stress, and a finite system cut out of an infinite Maxwell lattice must have zero modes as the perfect balance is disrupted by the missing links at edges. We note here that the same average coordination $z=3$ determines the critical point in the jamming of frictional disks with infinite friction coefficient [40-42]. This is not coincidental: the contact network at jamming also satisfies the Maxwell criterion [43], and the elements in Fig. 1(b) replicate the degrees of freedom and constraints between frictional disks in contact at the infinite friction limit. Just as the addition of friction changes the critical coordination at jamming from $z=4$ to $z=3$, the introduction of rotational degrees of freedom and shear constraints requires gear networks to be three-coordinated for topological mechanical modes to appear, unlike topological spring networks, which must have $z=4$ in two dimensions $[16,17,44]$.

Topological characterization.-For lattices made up of periodically repeating unit cells positioned at linear combinations of the primitive vectors $\mathbf{a}_{i}$, the Fourier-transformed extensions and displacements are related at each wave vector $\mathbf{k}$ in the Brillouin zone (BZ) via the relation $\mathbf{C}(\mathbf{k}) \mathbf{u}(\mathbf{k})=\mathbf{e}(\mathbf{k})$. Now, $\mathbf{C}(\mathbf{k})$ is a $2 n_{b} \times 3 n$ complex matrix for a unit cell with $n$ sites and $n_{b}$ links; it is a square matrix for Maxwell gear lattices with $2 n_{b}=3 n$. The index theorem, Eq. (1), applies at each point in the BZ. Since translations of the lattice along the $x$ and $y$ directions do not stretch or shear any links, there are at least two zero modes 
at $\mathbf{k}=0$, with two accompanying states of self-stress. However, for certain unit cell geometries, $\mathbf{C}(\mathbf{k})$ has no zero eigenvalues away from $\mathbf{k}=0$; we call this a gapped spectrum. Kane and Lubensky have recently shown [16] that square compatibility matrices with gapped spectra can be classified by a set of $d$ topological indices for $d$-dimensional lattices: the integer winding numbers $\left\{n_{1}, \ldots, n_{d}\right\}$ of the phase of $\operatorname{det} \mathbf{C}(\mathbf{k})=|\operatorname{det} \mathbf{C}(\mathbf{k})| e^{i \phi(\mathbf{k})}$,

$$
n_{i}=-\frac{1}{2 \pi} \oint_{C_{i}} d \mathbf{k} \cdot \nabla_{\mathbf{k}} \phi(\mathbf{k}),
$$

along the $d$ topologically distinct cycles $C_{i}$ of the BZ parallel to the reciprocal lattice directions $\mathbf{b}_{i}$ defined by $\mathbf{a}_{i} \cdot \mathbf{b}_{j}=2 \pi \delta_{i j}$. The topological nature of the $n_{i}$ is reflected in the fact that changing their values requires the spectral gap to close. The indices can be used to define a lattice vector $\mathbf{R}_{T}=\sum_{i} n_{i} \mathbf{a}_{i}$, called the topological polarization because it can be interpreted as a transfer of degrees of freedom along its direction, as we demonstrate.

To find Maxwell gear networks with a spectral gap and nontrivial topology [nonzero winding numbers of $\mathbf{C}(\mathbf{k})$ ], we consider modifications of the martini lattice [45], which has four sites and six links per unit cell on a regular hexagonal Bravais lattice (see Appendix B for descriptions of all lattices used in this work). By changing node positions and link lengths in the unit cell without affecting link connectivity, we find a range of distorted martini lattices with gapped spectra. Figures 2(a) and 2(c) show two examples. The lattice in Fig. 2(a) has $n_{1}=n_{2}=0$, whereas the lattice in Fig. 2(b) has $n_{1}=-1$ and $n_{2}=0$. As required, a one-parameter family of unit cells that connects the two lattices has a member with a gap closing in the spectrum of $\mathbf{C}(\mathbf{k})$, shown in Fig. 2(b). The compatibility matrix of this lattice has a line of zero eigenvalues extending through the $\mathrm{BZ}$ along $k_{x}=0$, so the winding numbers of $\operatorname{det} \mathbf{C}(\mathbf{k})$ become ill defined.

Edge mode polarization.-The winding numbers influence the count of zero modes at lattice edges [16]. Equation (1) dictates that a system cut out of an infinite Maxwell lattice will have an excess of zero modes equal to the number of deficient links, which is proportional to the length of the cut edges. When the compatibility matrix has a gapped spectrum, these zero modes cannot penetrate into the bulk (except for the two rigid-body translations at $\mathbf{k}=0$ ) and must therefore be localized at free surfaces [37,46]. As detailed in Appendix C, the count of zero modes localized at a free edge has two contributions: a local count $\nu_{L}$, which depends on the details of the edge termination, and a topological count $\nu_{T}$, which depends solely on the orientation of the edge normal relative to the bulk lattice polarization and is independent of local properties at the edge [16]. For example, the strip shown in Fig. 2(d) (with periodic boundary conditions along the vertical direction) has an identical number of missing constraints on the left and the right free edges, and the local count is $\nu_{L}=2$ per unit cell on both edges. However, the topological contributions are equal and opposite: $\nu_{T}=$ +2 and -2 on the left and right edges, respectively. The net count $\nu_{L}+\nu_{T}$ is four per edge unit cell on the left edge and zero on the right edge. The topological polarization $\mathbf{R}_{T}$ effectively transfers zero modes along its orientation, from the right edge to the left. A numerical calculation (Appendix D) confirms that all nontranslational zero modes are exponentially localized to the left edge, even for small separations between the left and right edges. The displacements and rotations associated with one such mode are visualized in Fig. 2(d).

To demonstrate the consequences of the topological bias in zero-mode distribution, we build a mechanical prototype of the polarized lattice with two free edges that run perpendicular to $\mathbf{R}_{T}$, as shown in Fig. 2(e). The topological polarization leads to a drastic asymmetry in stiffness between the left and right edges of the prototype, built from the metallic units shown in Fig. 1. As shown in Movie 1 of the Supplemental Material [47] and Fig. 2(e), the right edge and the bulk of the prototype are rigid under manual probing (aside from mechanical play), but the left edge can be deformed significantly from its original configuration. The topological character of the lattice governs the mechanical response of the prototype beyond the linear regime addressed by the mode calculation, giving rise to flexibility along a specific edge of an otherwise rigid structure. The polarization can also induce topological modes and states of self-stress at appropriately oriented domain walls separating lattices of different orientation, even if no bonds have been cut (Appendix E).

Topological Weyl modes.-In addition to modes localized at edges, Maxwell gear networks also support topologically protected zero modes and states of self-stress that extend into the bulk of a sample. These states, termed Weyl modes [17], are associated with gap closings at isolated "Weyl points" in the BZ away from $\mathbf{k}=0$ with nonzero windings $n_{w}=-\oint_{C} d \mathbf{k} \cdot \nabla_{\mathbf{k}} \phi(\mathbf{k}) /(2 \pi)$, where $C$ is a contour in momentum space encircling the point at which $\operatorname{det} \mathbf{C}(\mathbf{k})=|\operatorname{det} \mathbf{C}(\mathbf{k})| e^{i \phi(\mathbf{k})}=0$. Time-reversal symmetry ensures that $\mathbf{C}(\mathbf{k})$ and $\mathbf{C}(-\mathbf{k})$ share the same eigenvalues, which means that Weyl points always occur in pairs with equal and opposite windings. Weyl points and their associated Weyl modes can be removed only if pairs with opposite windings annihilate at high-symmetry points in the BZ. To realize such modes in geared metamaterials, we consider networks based on the hexachiral lattice [48], which has the same primitive vectors as the martini lattice and a 6-node, 9-link unit cell that forms hexagonal and triangular plaquettes [Fig. 5(b)]. The compatibility matrix of the regular hexachiral lattice has an ungapped spectrum, but distorting the plaquettes of the hexachiral lattice into irregular six-sided polygons can open up a spectral gap everywhere except at isolated points. An example is 
(a)

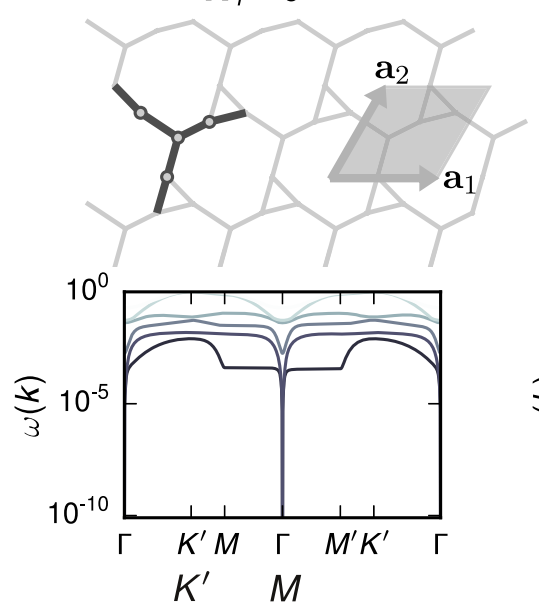

(b)
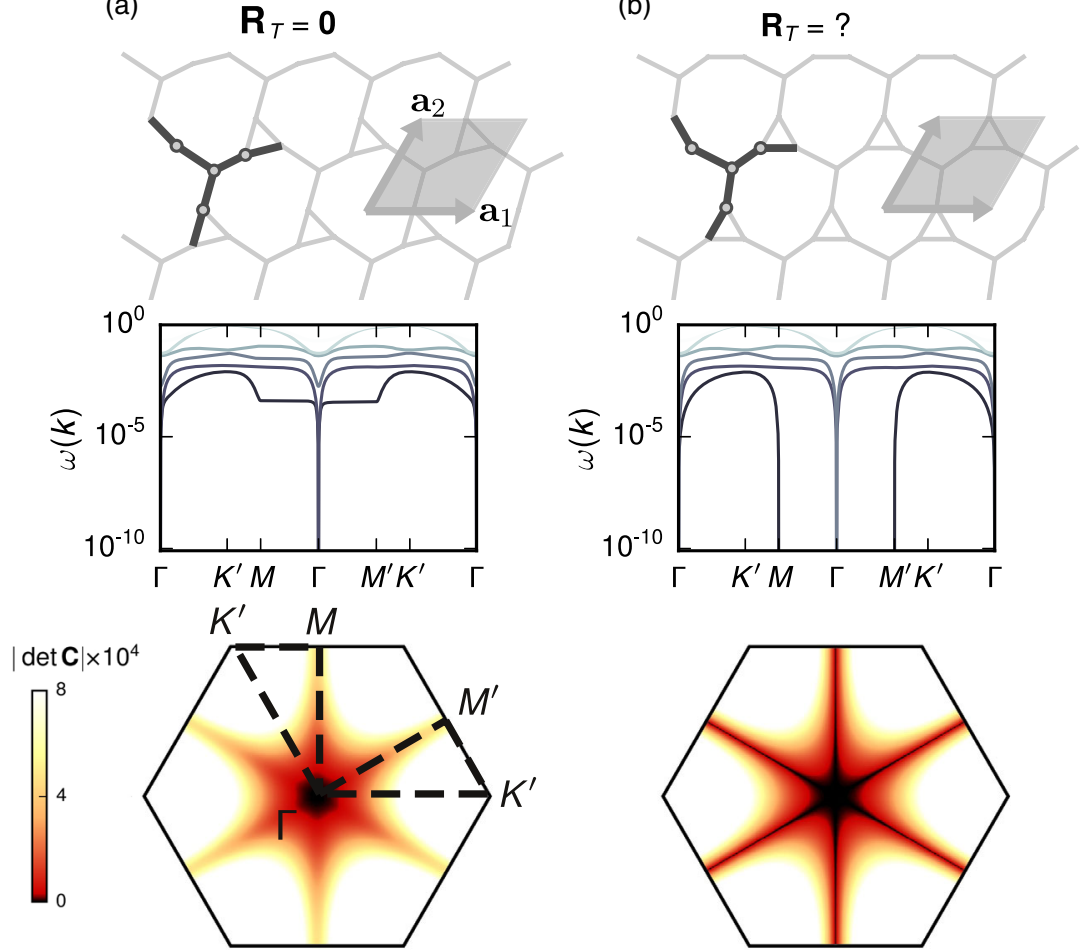

(c)
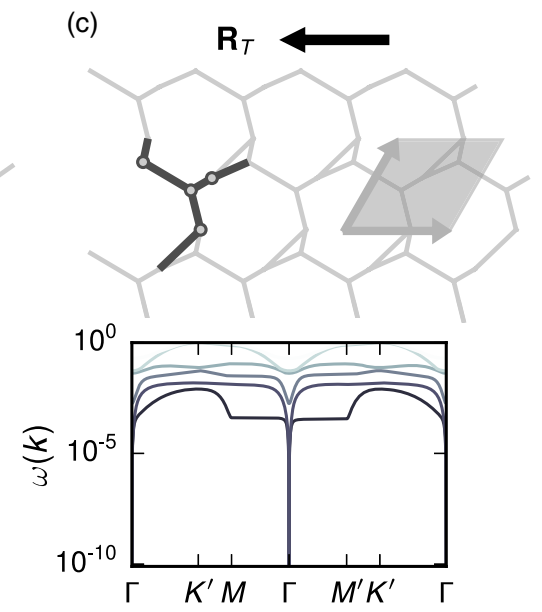

(d)

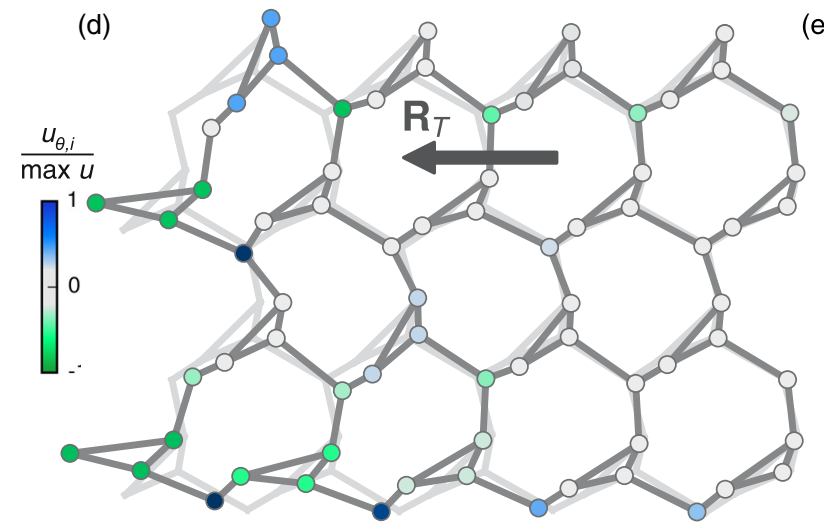

(e)

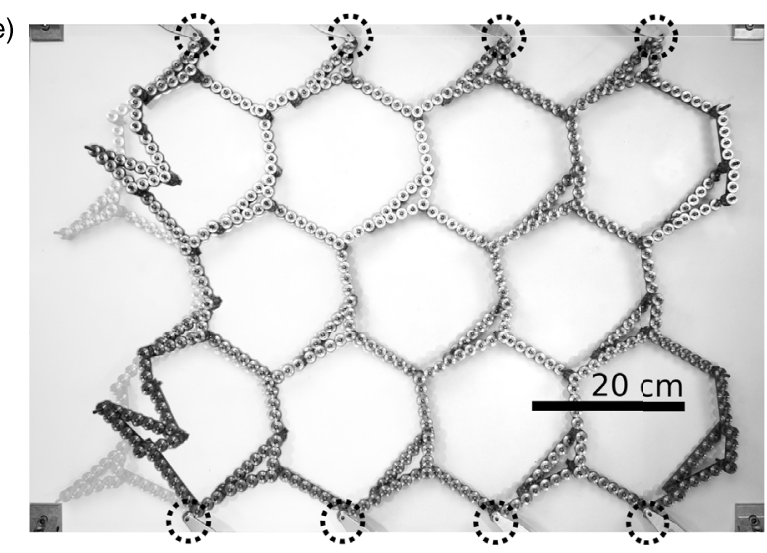

FIG. 2. Topologically polarized martini lattices. (a)-(c), Three members of a single-parameter family of martini lattices. The unit cell comprises four nodes (gray dots) and six unique links (thick black lines) and is repeated on a lattice built from primitive vectors $\left\{\mathbf{a}_{1}, \mathbf{a}_{2}\right\}$. Below each lattice are shown the determinant of the Fourier-transformed tension-shear compatibility matrix in the Brillouin zone (intensity plot) and the lowest six eigenvalues along specific contours (line plots). The lattices (a) and (c) have a gapped spectrum everywhere except at the $\Gamma$ point $(\mathbf{k}=0)$. The topological polarization, calculated as described in the text, is 0 in (a) and $-\mathbf{a}_{1}$ in (c). Lattice (b) has a gap closing along the line $k_{x}=0$, and the polarization is not well defined. (d) A strip of the polarized martini lattice with periodic boundary conditions along the $y$ direction and free boundaries on the left and right. Only the left edge localizes zero modes, one of which is visualized by node colors and displacements. (e), A physical prototype of the framework in (a) made out of elements illustrated in Fig. 1. The nodes along the top and bottom edges (encircled) are pinned down but free to rotate. The left edge has been deformed relative to the unperturbed lattice (overlay); no such deformations are possible at the right edge or in the interior.

shown in Fig. 3(b), which has gap closings at $\mathbf{k}= \pm(-0.3 / a, 3.0 / a)$, with nonzero winding numbers $n_{w}= \pm 1$. The periodic modulation of the corresponding topological Weyl modes is visualized in Figs. 3(d) and 3(e).

The structure shown in Fig. 3(b) is a member of a oneparameter family of distorted hexachiral lattices that smoothly interpolates between two lattices with gapped spectra and differing topological polarizations, shown in Figs. 3(a) and 3(c). Upon following this family of lattices, as is done in Movie 2 of the Supplemental Material [47], the gap first closes at the point $\mathbf{k}=(0,2 \pi / \sqrt{3} a)$ on the boundary of the BZ, which then splits into a pair of Weyl 
(a)

$\mathbf{R}_{T}=\mathbf{0}$
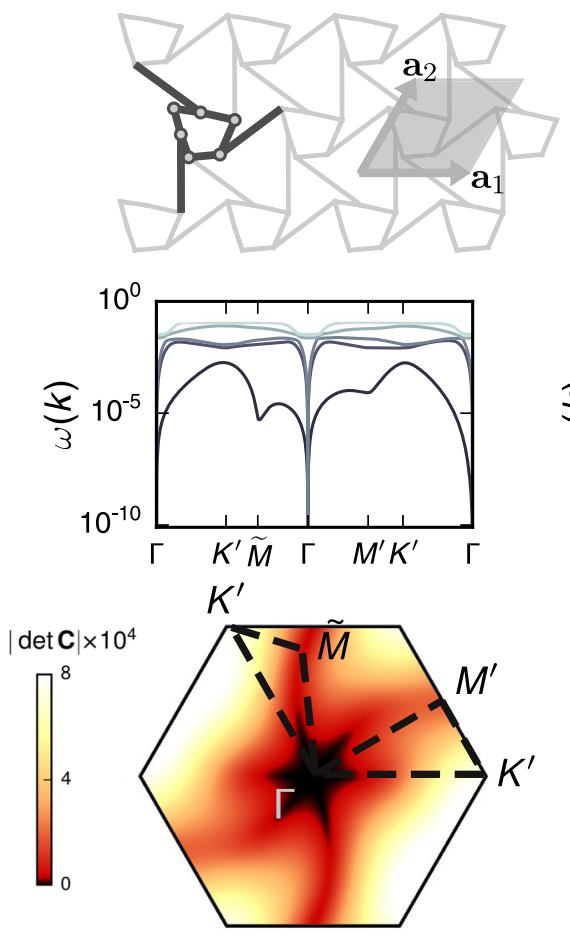

(d)

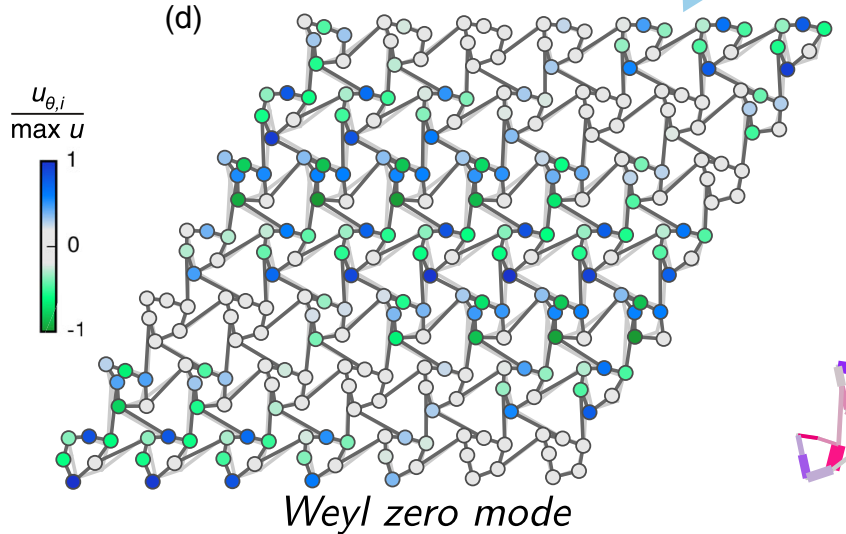

(b)

$\mathbf{R}_{T}=$ ?
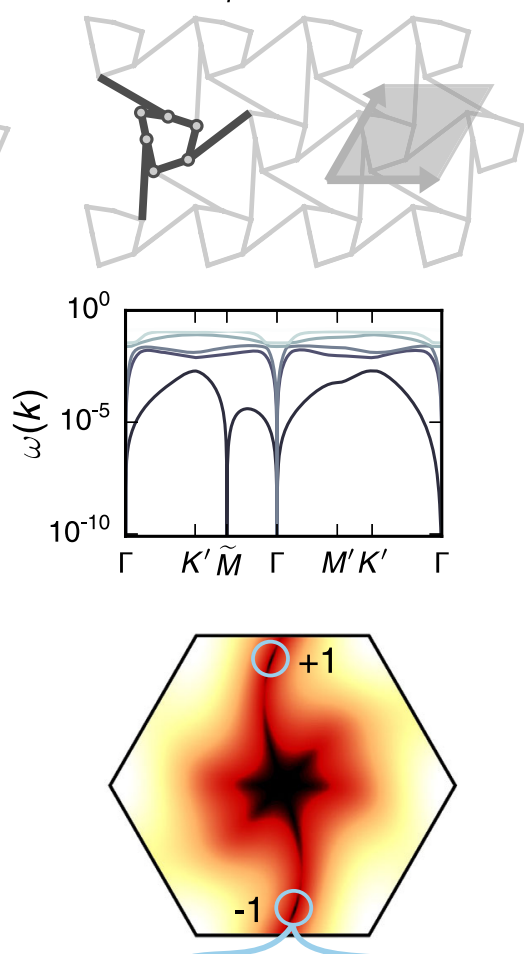

(c)
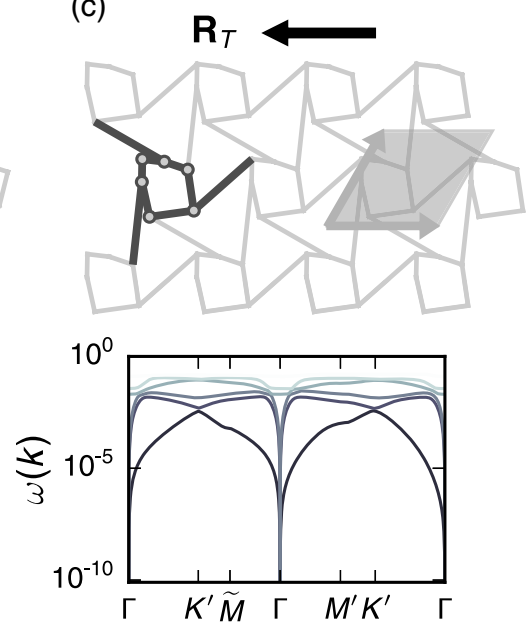

$(e)$
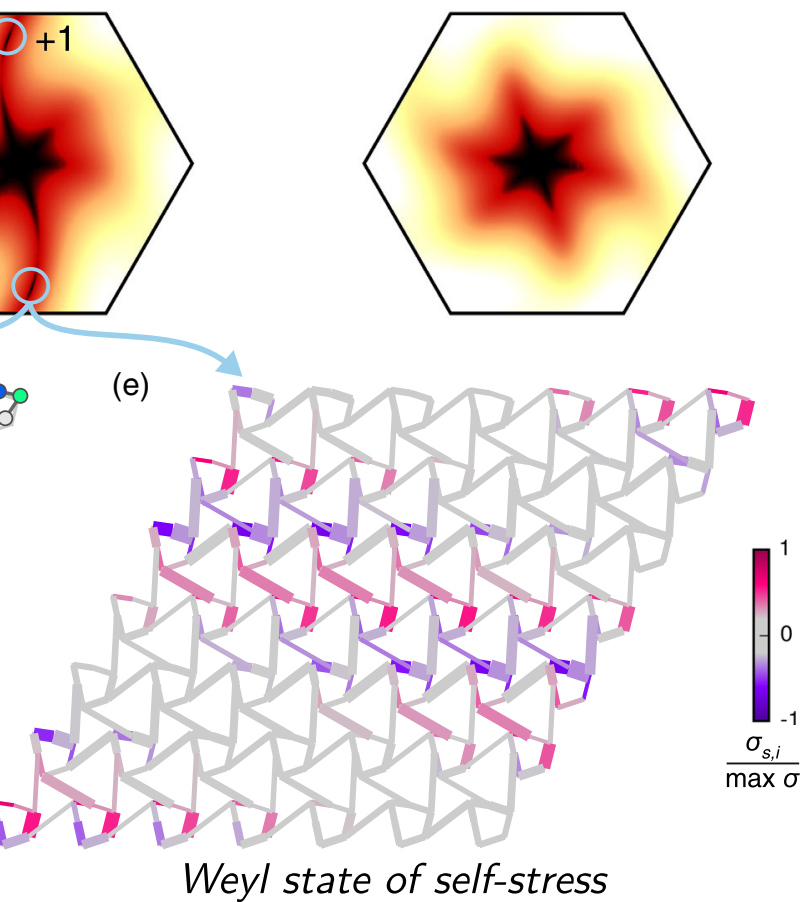

FIG. 3. Topological hexachiral lattices. (a)-(c) Three members of a single-parameter family of hexachiral gear lattices. The symmetric unit cell comprises six nodes (gray dots) and nine unique links (thick black lines). Below each lattice are shown the determinant of the Fourier-transformed tension-shear compatibility matrix in the BZ (intensity plot) and the lowest six eigenvalues along specific contours (line plots). The lattices (a) and (c) have a gapped spectrum everywhere except at $\mathbf{k}=0$. The topological polarization, calculated as described in the text, is 0 in (a) and $-\mathbf{a}_{1}$ in (c). Lattices (a) and (c) are separated by a family of lattices with a pair of Weyl points at which a zero eigenstate exists. One such lattice is shown in (b) with the Weyl points at $\mathbf{k}= \pm(-0.295 / a, 3.001 / a)$ encircled. The winding numbers $n_{w}= \pm 1$ are indicated. (d), (e) Visualization of the Weyl zero mode (d) and state of self-stress (e) at the lower Weyl point. The periodic variation of the modes is a consequence of the finite wave vector of the $\tilde{M}$ point at which the gap closes.

points with opposite winding numbers, $n_{w}= \pm 1$. The Weyl points sweep through the BZ, moving toward $\mathbf{k}=0$, where they annihilate. The change in the value of $\mathbf{R}_{T}$ between the gapped spectra in Figs 3(a) and 3(c) is effected by this traversal of Weyl points with nontrivial winding numbers across the BZ.

Guest-Hutchinson modes and global stability.-Periodic Maxwell lattices are perfectly balanced when degrees of freedom and constraints within the unit cell are considered. However, to build a periodic lattice, the primitive vectors $\mathbf{a}_{i}$ determining the relative placement of unit cells must also be specified. The two components for each primitive vector add up to four additional degrees of freedom. According to the index theorem, Eq. (1), the periodic lattice therefore has four excess zero modes when primitive vector changes are allowed. Three of these are the rigid-body transformations 
(two translations and a rotation) that do not deform any links. The remaining mode might either be decoupled completely from lattice displacements or correspond to an affine deformation that globally expands or shrinks the lattice, termed the Guest-Hutchinson mode [35]. All Maxwell spring lattices have a Guest-Hutchinson mode which, being a zero-energy affine deformation, leads to global collapse of the lattice under some external stresses [35-37]. The Guest-Hutchinson mode makes topological spring networks elastically unstable, but can be exploited to globally transform their topological properties [34].

The martini and hexachiral gear lattices differ drastically in their global mechanical stability when affine lattice deformations are taken into account. In the martini lattices, gear rotations are not allowed without node displacements since they are frustrated on the odd-sided plaquettes making up the lattice [Fig. 4(a)], and the nontrivial zero mode of the augmented compatibility matrix involves node
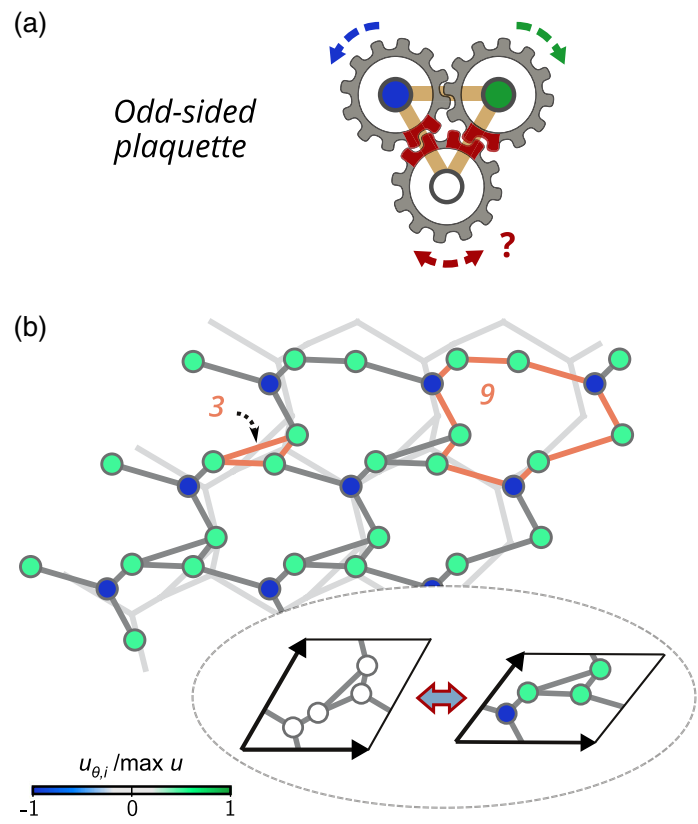

(c)

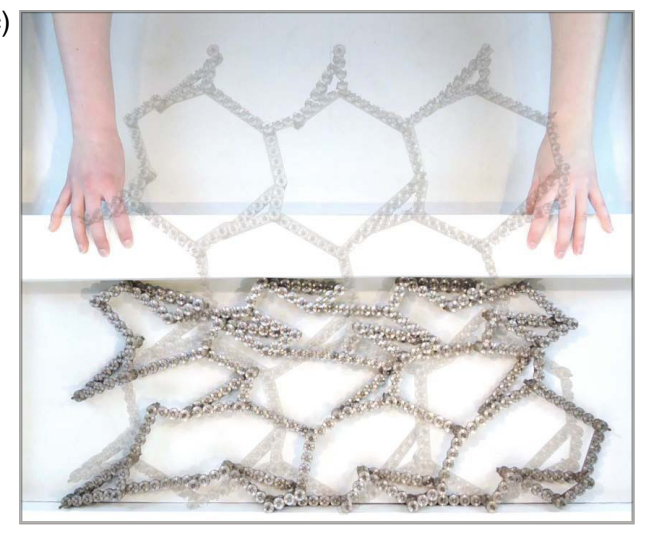

(d)

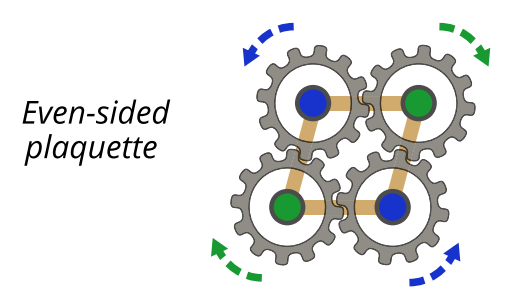

(e)
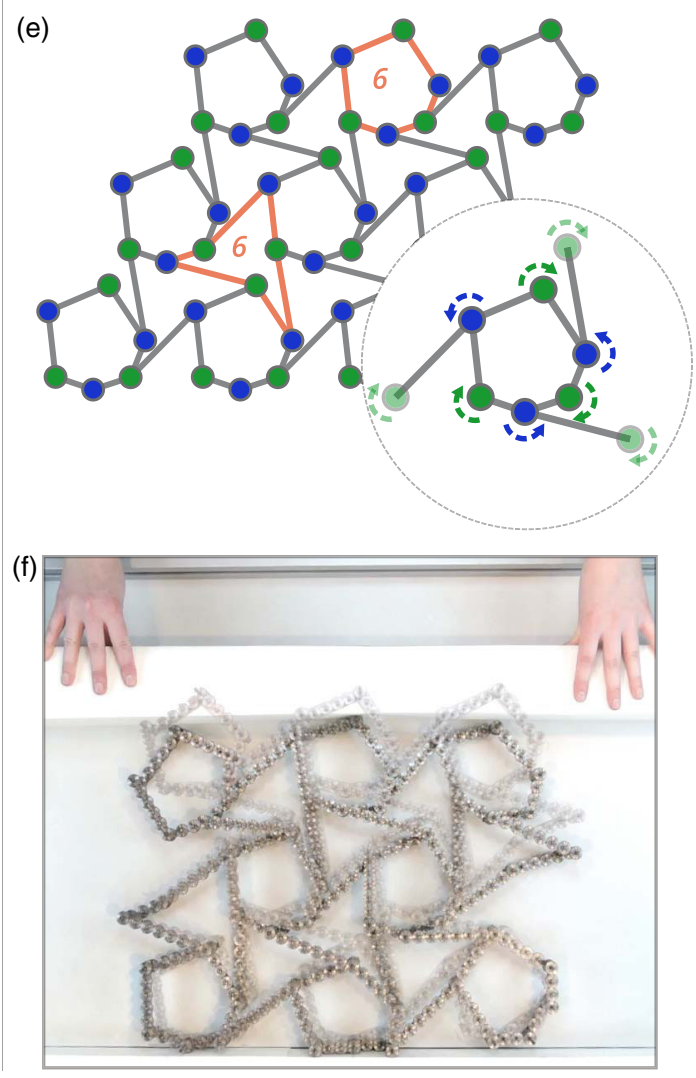

FIG. 4. Frustration and global deformations in martini and hexachiral lattices. Maxwell gear lattices with gapped spectra have a global zero-energy mode that differs qualitatively in the martini and hexachiral designs. (a) When an odd number of links is connected in a loop, rotation of gears without node displacements is frustrated. (b) The martini lattices have only odd-sided plaquettes with either 3 or 9 links (highlighted). Therefore, the zero-energy mode (visualized in the overlay) is a Guest-Hutchinson mode that requires point displacements as well as a distortion of the unit cell. The zoom shows the zero-energy deformation of the unit cell, including the change in primitive lattice vectors. (c) A prototype martini lattice with free boundaries cannot resist vertical compression (horizontal bar being pushed down) because of the Guest-Hutchinson mode (the precompression configuration is shown in overlay). (d) Gears on an evensided loop of links can counterrotate freely without node displacements. (e) A hexachiral lattice consists solely of six-sided plaquettes (highlighted). It can be divided into two sublattices, with all gears rotating counterclockwise (blue) on one sublattice and clockwise (green) by the same amount on the other without frustration. This is the required global zero-energy mode of the periodic system, which does not displace any nodes. (f) The absence of a unit-cell-shape changing mode imparts stability to the hexachiral lattice, which largely maintains its shape under uniform compression. 
displacements and a change in primitive vectors depicted in Fig. 4(b). As a result, the martini gear lattice is unstable against uniform compressions along the vertical direction [see Movie 3 of Supplemental Material [47] and Fig. 4(c)].

By contrast, hexachiral gear lattices avoid the nonlinear collapse mode by taking advantage of rotational degrees of freedom. Unlike the martini lattices, they are composed solely of even-sided plaquettes, which support gear counterrotations without node displacements. The two-color theorem of graphs [49] ensures that such lattices are bipartite, i.e., divisible into two sublattices so that no two nodes in the same sublattice are linked. As a result, they harbor a global zero-energy mode at $\mathbf{k}=0$, in which gears on the two sublattices counterrotate without hindrance [50], as shown in Fig. 4(e). These collective rotations absorb the Guest-Hutchinson mode, eliminating the global collapse mechanism. Consequently, gapped hexachiral gear lattices are stable against external loads [Fig. 4(f)]. Note that the lattice is unstable against torques applied on individual gears (which may drive the global counterrotation mode without resistance), but this will not lead to shape-changing deformations.

Towards geared metamaterials.-We now comment on practical considerations in implementing the proposed designs. The topological mechanisms are independent of the size of the building blocks, as long as the relevant degrees of freedom and constraints (Fig. 1) are incorporated. At architectural or tabletop scales, the designs may be realized by assembling beams and gears manually, as demonstrated by our prototypes, but the number of repeating units is limited by practical constraints on time and effort. The number of gears needed per unit cell could be dramatically reduced if gears of multiple diameters are used. To increase the number of unit cells, however, the assembly process itself must be automated. Modern additive manufacturing techniques allow multicomponent assemblies with free [51] or lubricated [52] joints to be $3 \mathrm{D}$ printed in one shot, providing a platform to scale up the designs here to structures with hundreds or thousands of unit cells while bringing down the size of the individual units to microscopic scales.

Construction may be simplified by using gear-free elements that approximate the required distance and shear constraints. In Appendix F, we describe an assembly of four freely hinged beams that reproduces the constraints of the gear link to linear order in node displacements and rotations and, therefore, generates the same entries in the compatibility matrix. A structure built out of these elements would display the same linear excitations (including zero-energy modes) as the gear network with fewer moving parts, but its nonlinear response at large deformations would differ. The hinged beams may be implemented as continuous elements with slender flexible joints [13], enabling fabrication via standard additive manufacturing techniques over a wide range of length scales.
Regardless of fabrication technique, real structural elements are liable to incorporate additional degrees of freedom (e.g., mechanical backlash or play) and constraints (e.g., finite friction of gear shafts) beyond those considered by the model. As is true for any metamaterial design, the predicted behavior will be observed as long as the desired degrees of freedom and constraints dominate the spurious ones for relevant loads. For instance, if the components are sufficiently rigid, the torque needed to overcome shaft friction is associated with a negligibly small deformation of the gears compared to the desired motion. Our prototypes demonstrate that this separation is achievable in practice. Unconstrained play is clearly distinguishable from true mechanisms, which involve coordinated node motion and gear rotation (see Movie 1 of the Supplemental Material [47]). Manually actuating the mechanisms requires some effort to overcome substrate and gear-shaft friction, but the forces involved are negligible compared to those needed to significantly deform the metal links.

The topological nature of the zero-energy modes ensures their protection against a large range of structural imperfections. For example, the bias in edge mechanisms is predicted by the topological polarization [Figs. 2(d) and 2(e)] for a periodic lattice in the bulk, but is guaranteed to persist for any perturbation of the interior points as long as the perturbation does not close the bulk phonon gap. Such a gap closing would correspond to the appearance of zeroenergy modes that extend from the left edge to the right edge of the system, requiring large coordinated global changes of the unit cells. As long as the chosen unit cell is far from singular gap-closing configurations, such as the lattice in Fig. 2(b), perfect crystalline order is not required for the topological bias in zero modes to persist. Similarly, local defects in the structure, such as slipped, jammed, or missing gears, may introduce localized zero modes or states of self-stress, but their influence on localized boundary modes is exponentially small in their distance from the boundary. Defects on or near the left boundary in Fig. 2(d) may add or remove individual zero modes, but cannot change the overall flexibility of the edge, which arises from a macroscopic number (proportional to the length of the edge) of topological modes.

Conclusion.-We design and build topological geared metamaterials in which translational and rotational degrees of freedom are constrained in a geometrically controlled manner giving rise to tunable mechanical properties. Gear lattices satisfying the Maxwell criterion for marginal rigidity support zero modes and stress states of topological origin like their spring lattice counterparts, but with the additional ability to independently tune the global elastic stability. The absence of a zero-energy collapse mode in certain unfrustrated gear lattices, which is a direct consequence of the rotational degrees of freedom, paves the way towards building topological mechanical structures that maintain their shape without requiring extra constraints 
such as pinning to a static background. The key insight, that expanded degrees of freedom and interactions that go beyond harmonic springs open up new possibilities for topological mechanics, is applicable to a wide range of systems, including colloidal or molecular assemblies that interact via noncentral forces.

\section{ACKNOWLEDGMENTS}

We thank the Leiden University Fine Mechanics Department for technical support, and Bryan Chen and Anton Souslov for useful discussions. This work was funded by FOM, a Delta ITP Zwaartekracht grant, and a VIDI grant from NWO.

A. S. M. and J. P. contributed equally to this work.

\section{APPENDIX A: COMPATIBILITY MATRIX FOR GEAR ELEMENTS}

The compatibility matrix $\mathbf{C}$ of a gear lattice is built up of contributions from individual links. Each constraint adds a row to the matrix and each degree of freedom is assigned to a column. The contribution of link $m$ of length $L$ which connects nodes $i, j$ is given by [53]

$$
\left(\begin{array}{c}
e_{l, m} \\
e_{s, m}
\end{array}\right)=\left(\begin{array}{cccccc}
\frac{L_{x}}{L} & \frac{L_{y}}{L} & 0 & -\frac{L_{x}}{L} & -\frac{L_{y}}{L} & 0 \\
-\frac{L_{y}}{L} & \frac{L_{x}}{L} & \frac{L}{2} & \frac{L_{y}}{L} & -\frac{L_{x}}{L} & \frac{L}{2}
\end{array}\right)\left(\begin{array}{c}
u_{x, i} \\
u_{y, i} \\
u_{\theta, i} \\
u_{x, j} \\
u_{y, j} \\
u_{\theta, j}
\end{array}\right) .
$$

Here, $L_{x}$ and $L_{y}$ are the projections of the link's length along $\hat{x}$ and $\hat{y}, e_{l, m}$ and $e_{s, m}$ represent the tension and shear strains on the link, and $u_{x, i}, u_{y, i}$ and $u_{\theta, i}$ denote the translational and rotational displacements of the $i$ th node.

Therefore, each link in an assembly provides two rows to the compatibility matrix. The number of columns expands to the degrees of freedom in the assembly, with zero entries for degrees of freedom unassociated with the link that contributes a particular row.

For periodic lattices, the Fourier-transformed compatibility matrix $\mathbf{C}(\mathbf{k})$ has one row (column) for each constraint (degree of freedom) in the unit cell. Row entries for links that connect nodes in different unit cells separated by a Bravais lattice vector $R$ acquire a complex phase factor $e^{i \mathbf{k} \cdot \mathbf{R}}$.

\section{APPENDIX B: UNIT CELL DEFINITIONS FOR PERIODIC LATTICES}

The three-coordinated Maxwell lattices we consider are based on the martini lattice, originally developed to study
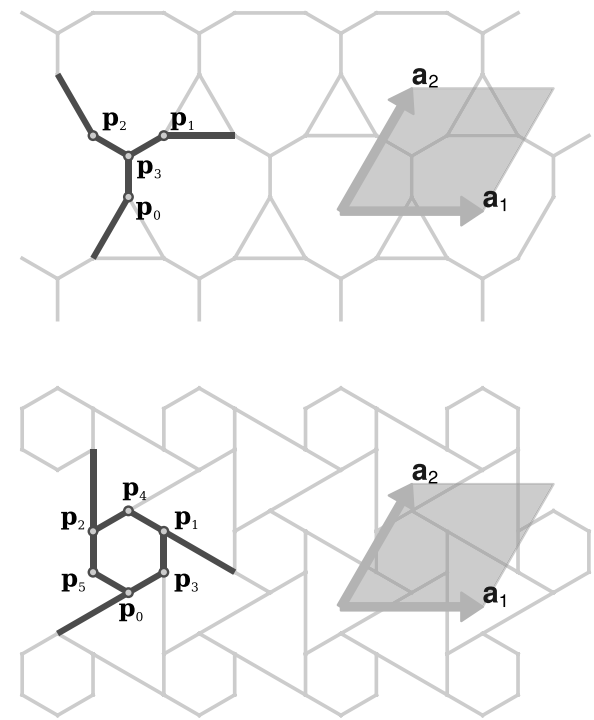

FIG. 5. Regular martini (top) and hexachiral (bottom) lattices. Symmetric unit cells are shown with points labeled.

percolation [45], and the hexachiral lattice, which formed the basis of an early negative Poisson ratio metamaterial [48]. Figure 5 shows the unit cells for the regular martini and hexachiral lattices. The lattices use the same underlying hexagonal Bravais lattice, with primitive vectors $\mathbf{a}_{1}=a \hat{x}$, $\mathbf{a}_{2}=(a / 2) \hat{x}+(\sqrt{3} a / 2) \hat{y}$. The martini lattice has four nodes and six unique links per unit cell, whereas the hexachiral unit cell has six nodes and nine unique links.

The lattices we study in the main text are completely defined by specifying the positions of the nodes labeled in Fig. 5. Tables I and II provide definitions of the distorted martini and hexachiral lattices, respectively.

\section{APPENDIX C: TOPOLOGICAL POLARIZATION AND ZERO-ENERGY EDGE MODES}

The formalism for counting the number of net degrees of freedom (i.e., number of zero modes minus the number of states of self-stress) localized to a particular edge of a Maxwell lattice with a gapped spectrum was developed in Ref. [16] (see also Ref. [37]). In this Appendix, we summarize the main results in the context of gear lattices.

As explained in the main text, free edges of Maxwell lattices are associated with zero-energy modes. There are two contributions to the number of zero modes per repeating unit along the edge. The first is a local count of the missing links along the edge, whose computation can be explained using an electrostatic analogy. Upon associating "charges" of +3 (for the three degrees of freedom) to each node and -2 (for the two constraints) to the center of each link, a Maxwell lattice has a charge-neutral unit cell, but a dipole moment [16] can be defined as

$$
\mathbf{d}=3 \sum_{i} \mathbf{r}_{i}-2 \sum_{m} \mathbf{r}_{m}
$$


TABLE I. Unit cell descriptions of distorted martini lattices. Labels refer to the lattices in Fig. 2. The position of the nodes [labeled in Fig. 5(a)] is specified in units of the lattice constant $a$; the primitive vectors and link connectivity are unchanged.

\begin{tabular}{ll}
\hline \hline Lattice & Node positions \\
\hline A & $\mathbf{p}_{0}=(-0.05,-0.38), \mathbf{p}_{1}=(0.35,0.15), \mathbf{p}_{2}=(-0.30,0.23), \mathbf{p}_{3}=(0.05,-0.00)$ \\
$\mathrm{B}$ & $\mathbf{p}_{0}=(0.00,-0.38), \mathbf{p}_{1}=(0.33,0.19), \mathbf{p}_{2}=(-0.33,0.19), \mathbf{p}_{3}=(0.05,-0.00)$ \\
$\mathrm{C}$ & $\mathbf{p}_{0}=(0.15,-0.38), \mathbf{p}_{1}=(0.25,0.11), \mathbf{p}_{2}=(-0.40,0.27), \mathbf{p}_{3}=(0.05,-0.00)$ \\
\hline \hline
\end{tabular}

TABLE II. Unit cell descriptions of distorted hexachiral lattices. Labels A-C refer to the lattices in Fig. 3, and label D is the lattice used in Fig. 4. The position of the nodes [labeled in Fig. 5(b)] is specified in units of the lattice constant $a$; the primitive vectors and link connectivity are unchanged.

\begin{tabular}{ll}
\hline \hline Lattice & \multicolumn{1}{c}{ Node positions } \\
\hline $\mathrm{A}$ & $\mathbf{p}_{0}=(-0.14,-0.04), \mathbf{p}_{1}=(0.22,-0.22), \mathbf{p}_{2}=(0.04,0.17), \mathbf{p}_{3}=(-0.07,-0.25), \mathbf{p}_{4}=(0.36,0.10), \mathbf{p}_{5}=(-0.20,0.20)$ \\
$\mathrm{B}$ & $\mathbf{p}_{0}=(-0.15,-0.04), \mathbf{p}_{1}=(0.23,-0.23), \mathbf{p}_{2}=(0.04,0.17), \mathbf{p}_{3}=(-0.09,-0.33), \mathbf{p}_{4}=(0.31,0.08), \mathbf{p}_{5}=(-0.20,0.20)$ \\
$\mathrm{C}$ & $\mathbf{p}_{0}=(-0.17,-0.04), \mathbf{p}_{1}=(0.31,-0.31), \mathbf{p}_{2}=(0.04,0.14), \mathbf{p}_{3}=(-0.10,-0.36), \mathbf{p}_{4}=(0.25,0.07), \mathbf{p}_{5}=(-0.16,0.16)$ \\
$\mathrm{D}$ & $\mathbf{p}_{0}=(-0.44,-0.20), \mathbf{p}_{1}=(0.07,-0.21), \mathbf{p}_{2}=(-0.08,0.42), \mathbf{p}_{3}=(-0.19,-0.29), \mathbf{p}_{4}=(0.17,0.05), \mathbf{p}_{5}=(-0.49,0.24)$ \\
\hline \hline
\end{tabular}

where $\mathbf{r}_{i}$ and $\mathbf{r}_{m}$ are, respectively, the position vectors of node $i$ and link $m$ within the unit cell. At a particular edge, a unit cell must be chosen that is compatible with the edge termination (with no dangling bonds), leading to a nonzero edge-specific dipole moment $\mathbf{R}_{L}$. The local count due to missing constraints is determined by evaluating the surface charge due to the dipole moment of the surface unit cell: $\nu_{L}=\mathbf{G} \cdot \mathbf{R}_{L} / 2 \pi$, where $\mathbf{G}$ is the reciprocal lattice vector that points outward from the edge.

The second contribution is the topological count, which is determined by the properties of the gapped bulk away from the edge. The form of the compatibility matrix depends on the choice of unit cell; to calculate bulk properties such as the winding numbers $n_{i}$, a natural choice is a balanced unit cell with $\mathbf{d}=0$, such as the unit cells highlighted in the first rows of Figs. 2 and 3. For lattices with one or more nonzero winding numbers $n_{i}$, additional zero modes arise from the existence of the bulk topological invariant $\mathbf{R}_{T} \neq 0$, which induces $\nu_{T}=\mathbf{G} \cdot \mathbf{R}_{T} / 2 \pi$ modes per edge unit cell [16]. $\mathbf{R}_{T}$ is an extra dipole moment contribution that is not apparent purely from geometric considerations of placing nodes and links as in Eq. (C1), and is therefore termed a topological polarization. For a section with two parallel edges, such as in Fig. 2(d), G flips sign from one edge to the other, whereas $\mathbf{R}_{T}$ is a bulk property. The topological mode count is equal and opposite on the edges, analogous to a transfer of "charge" (i.e., degrees of freedom) from one edge to the other along the polarization direction.

We now calculate the zero-mode count at each edge of the strip of the polarized martini lattice in Fig. 2(d) with periodic boundary conditions along the vertical direction and free edges on the left and right. The left free edge, which runs perpendicular to the reciprocal lattice vector
$\mathbf{G}=-4 \pi \hat{x} / a$, carries an edge dipole moment $\mathbf{R}_{L}{ }^{1}=-\mathbf{a}_{1}$. The total number of zero modes per unit cell along that edge is $\mathbf{G}^{1} \cdot\left(\mathbf{R}_{T}+\mathbf{R}_{L}{ }^{1}\right) / 2 \pi=4$. Figure $2(\mathrm{~d})$ shows one such local zero mode, numerically calculated assuming periodic boundary conditions along the vertical direction. Conversely, the right free edge has $\mathbf{R}_{L}^{2}=\mathbf{a}_{1}=-\mathbf{R}_{T}$, and the net mode count is zero. Although the nodes along the edge are missing links relative to the interior, the local and topological contributions to the unconstrained degrees of freedom cancel. This asymmetry in the linear zero-mode count translates to a drastic difference in rigidity between the left and right edges of the prototype probed in Fig. 2(e) and Movie 1 of the Supplemental Material [47].

\section{APPENDIX D: LOCALIZATION AND SIZE DEPENDENCE OF EDGE MODES IN FINITE SAMPLES}

Appendix $\mathrm{C}$ describes the local and topological count of zero modes or states of self-stress exponentially localized to an edge of a semi-infinite system. For example, the left edge of the strip shown in Fig. 2(d) is predicted to have four zero modes per repeating unit in isolation, whereas the right edge has no zero modes in isolation. In this situation, however, the conclusions hold even for a strip of finite width, where the left and right edges are separated by only a few lattice spacings. To understand this, we note that a zero mode is a set of displacements and rotations that does not violate any link constraints. If this is true for a semi-infinite system, it is also true for a subsection cut out of that system, because the displacements do not rely on any stresses being borne by the cut links. Therefore, zero modes of a semiinfinite system are also zero modes of the finite-width strip. Since all the zero modes are localized to the left edge, they 
remain so even when the right edge is brought close to the left edge.

To illustrate this, we numerically study the spatial structure of the topological zero modes for the strip in Fig. 2(d), which are the zero eigenvectors of the dynamical matrix $\mathbf{D}=\mathbf{C}^{\mathrm{T}} \mathbf{C}$ [we set all constraint stiffnesses or spring constants to 1]. The free left and right edges are obtained by removing four links from a periodic system, so the compatibility matrix has eight fewer rows than columns. Its null space, which is also the null space of $\mathbf{D}$, contains eight independent vectors, which are the zero modes of the structure. Two of these are rigid-body translations along $x$ and $y$; we remove the corresponding displacement vectors from the null space to obtain six independent null vectors with no translation component. Figure 6 shows the displacement amplitude averaged along the vertical direction as a function of distance from the left edge for these six modes. All modes decay exponentially as a function of distance from the left edge, with a decay length of about two lattice spacings, and the mode amplitudes are diminished by an order of magnitude at the right edge, even for a strip that is just four lattice units wide. The right edge does not acquire new zero modes in the finite system, because the index theorem [Eq. (1)] constrains the total number of zero modes minus stress states for the missing links, and all eight excess zero modes are accounted for. The right edge could acquire new zero modes only if states of self-stress arose elsewhere in the system to keep $n_{m}-n_{\mathrm{ss}}=8$ constant.

We note that the argument does not hold for states of self-stress: a state of self-stress localized to an edge of a semi-infinite system is not automatically a state of selfstress of a subsystem cut out of it, because it might rely on some of the cut links to maintain equilibrium.

The physical prototype, Fig. 2(e), does include additional constraints because the top and bottom points have been pinned. Therefore, some of the zero modes associated with the left edge will acquire a small stiffness. Nevertheless, the additional constraints cannot make the right edge soft, and the asymmetry of stiffness between left and right edges persists in the presence of pinning, as is observed upon manually probing the edges (see Movie 1 of Supplemental Material [47]).

\section{APPENDIX E: ZERO MODES AND STATES OF SELF-STRESS AT DOMAIN WALLS}

Besides influencing the count of zero modes at lattice edges, the topological polarization also induces zero modes and states of self-stress at domain walls separating lattices of different polarizations, even though no links have been cut or added relative to a perfectly balanced (isostatic) system. Reference [16] showed that, when a region $S$ of a system has no missing bonds (i.e., all points satisfy the Maxwell criterion), then the "topological count" of the number of zero modes minus the number of states of selfstress in the interior of the region is given by the net flux of polarization through its boundary: in two dimensions, $\nu_{T}=\left(1 / A_{\text {cell }}\right) \int_{\partial S} \mathbf{R}_{T} \cdot \hat{n} d S$, where $A_{\text {cell }}$ is the area of a unit cell and $\hat{n}$ is the inward boundary normal of the boundary $\partial S$, which is required to lie in a region with a spectral gap (i.e., there are no zero modes or states of selfstress on the boundary of the region).
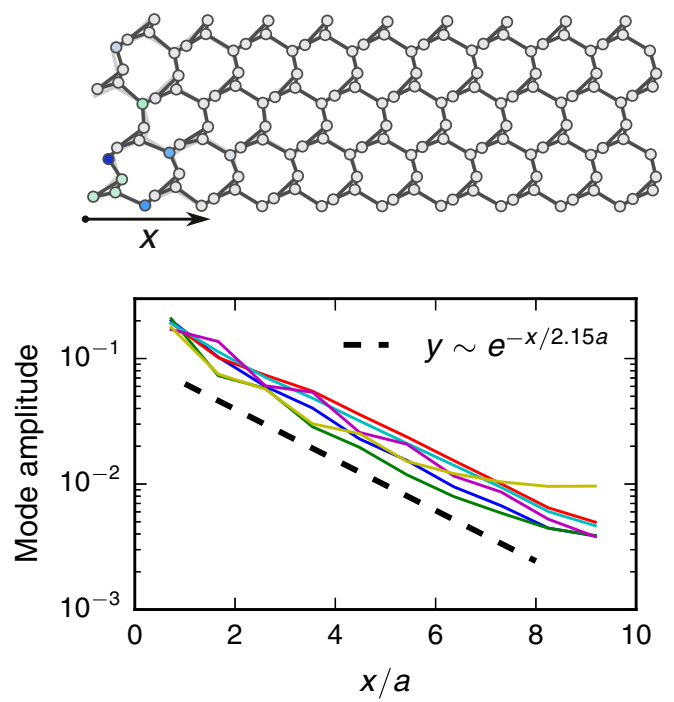

FIG. 6. Decay of displacement amplitudes for nontranslational zero modes as a function of distance from the left edge for systems with free boundaries perpendicular to the $x$ direction and periodic boundary conditions along $y$. Numerically, eight zero modes are found as predicted by the index theorem. Two of these are the uniform translations, while the remaining six are shown to decay exponentially from the left edge with a decay length comparable to the lattice spacing $a$. The dashed line is a fit of the sum of the mode amplitudes to a simple exponential decay. Left: System shown in Fig. 2(d). Right: System with twice the separation between the free left and right edges. 
Consider the domain wall geometry in Fig. 7(a), with periodic boundary conditions along both directions so that every node has exactly three links. Two domain walls (dashed lines) separate a region of the polarized martini lattice from a region of the same lattice rotated by $\pi \mathrm{rad}$, which also rotates the topological polarization. Therefore, a region surrounding the left domain wall has a net influx of polarization, whereas a region surrounding the right domain wall has a net outflux. Calculating the fluxes, we expect eight zero modes exponentially localized to the left domain wall and eight states of self-stress localized to the right domain wall. However, because the separation

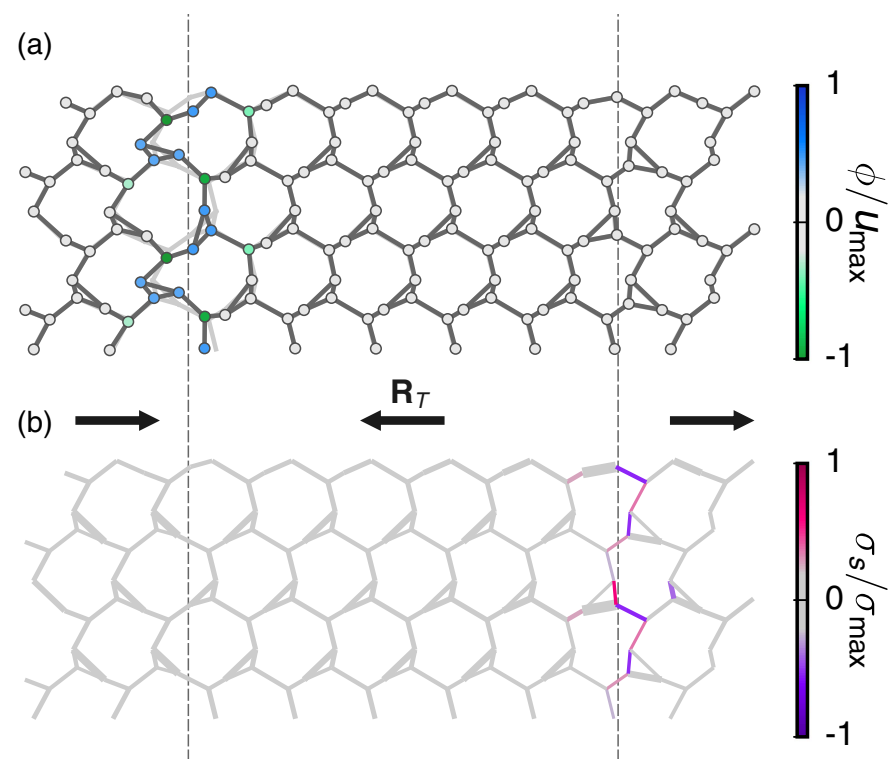

(c)

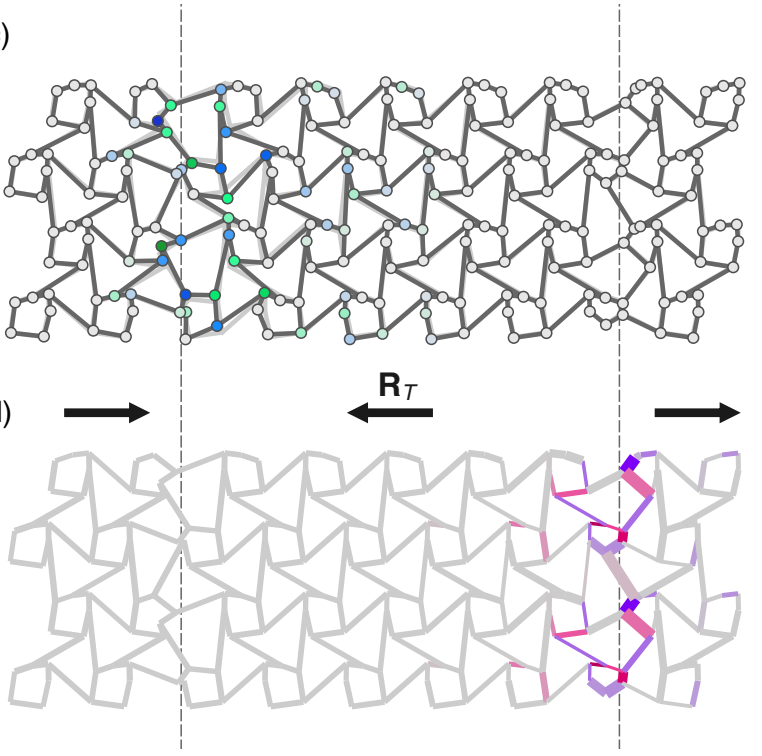

(e)

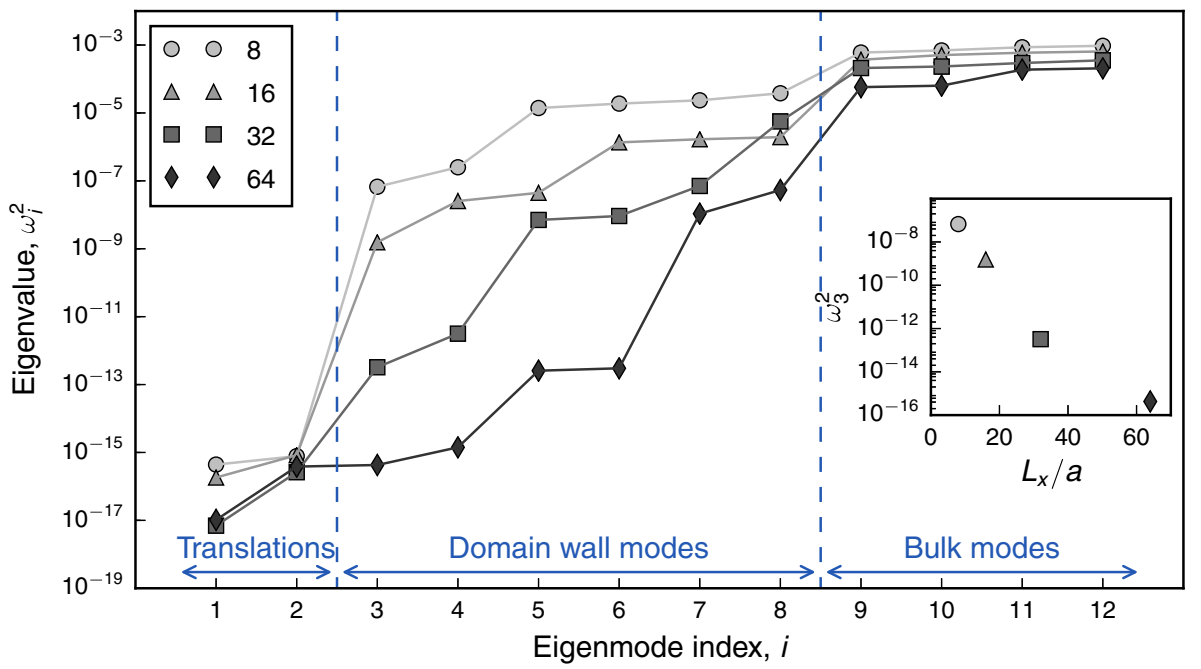

FIG. 7. Topological modes and states of self-stress at domain walls. (a),(b) Domain walls (dotted lines) separating oppositely oriented sections of the polarized martini lattice. Although the number of degrees of freedom and constraints are unchanged along the domain walls, they localize zero-energy modes and stress states because they carry a net polarization flux. The domain wall on the left has a net influx of polarization and localizes zero modes. In finite systems, these become soft modes and stress states with energy exponentially small in system size, one of which is visualized in (a). The dark lines show a lattice with nodes moved in proportion to the displacement components of the zero eigenvector, and colored by the torsional components. The light gray lines show the undeformed lattice for comparison. The domain wall on the right has a net outflux of polarization and localizes states of self-stress, one of which is visualized in (b). Bonds are colored by the shear components of the zero stress eigenvector, and their thicknesses are proportional to the tension components. (c),(d) Same as in (a),(b) for the polarized deformed hexachiral lattice. (e) System size dependence of the localized mode energies. The lowest eigenvalues of the dynamical matrix are shown as a function of system length along the horizontal direction for the domain wall geometry of (a) and (b), with domain walls always placed $0.66 L_{x}$ apart. Inset: Eigenvalue of the first nontranslation mode (index $i=3$ ) as a function of system size. 
between the domain walls is finite, the boundaries of each region do not lie in fully gapped parts of the lattice because there are components of the topological modes from the other domain wall in the same part, albeit exponentially small in the distance from the other domain wall. Therefore, we expect corrections to the energies of the localized modes that fall exponentially with system size.

To test the dependence of the mode energies on system size, we numerically compute the lowest eigenvalues of the dynamical matrix for systems with different lengths ranging from $L_{x}=8$ to 64 unit cells. In each case, the domain wall separation is $0.66 L_{x}$. The results [Fig. 7(e)] show that the lowest eight eigenvalues have a qualitatively different system size dependence than the higher eigenmodes. Rigidbody translations always make up the lowest two modes (which are zero energy up to numerical precision), but the next six modes have significant weights on the left domain wall. Above eigenmode 9, the modes have the form of long-wavelength plane waves, delocalized into the bulk. The energies of the domain wall modes fall sharply with system size compared to the bulk modes, and at the larger system size the two lowest-energy domain wall modes are indistinguishable in energy from the rigid-body translations. The inset of Fig. 7(e) shows the exponential fall in eigenvalue with system size, consistent with the expected finite-size correction to the mode energy.

\section{APPENDIX F: ALTERNATIVE LINK DESIGN}

The gear network we discuss in the main text deviates from spring network models. Spring networks consist of freely pivoting nodes, connected by springs that provide tensile constraints between them. By contrast, pivoting node motions are constrained in the geared network, so that only equal and opposite rotations are allowed at each pair of adjacent nodes. We propose an alternative link design that replicates this constraint on nodal rotations in the linear regime, without the use of gears.

The alternative link design is shown in Fig. 8(a). The bars in the link are assumed to resist stretching, shearing, and bending, while all nodes may pivot freely. In this linking mechanism, the polar angle $\alpha_{i}=\alpha_{0}$ at node $n_{i}$ may be increased to an angle $\alpha_{i}=\alpha_{0}+\delta \alpha_{i}$. As a result, the polar angle $\alpha_{j}=\pi+\alpha_{0}$ at node $n_{j}$ increases to $\alpha_{j}=\pi+\alpha_{0}+\delta \alpha_{j}$. The relation between $\delta \alpha_{i}$ and $\delta \alpha_{j}$ is given in the linear regime by

$$
\delta \alpha_{j}=-\delta \alpha_{i},
$$

which is obtained as follows. Assuming no deformations in the beams,

$$
\left|\vec{p}_{1}-\vec{p}_{2}\right|^{2}=l^{2},
$$

where $l$ is the length of the subbeam connecting subnodes $p_{1}$ and $p_{2}$, holds for any angles $\alpha_{i}, \alpha_{j}$ compatible with the link's geometry. Now, (a)

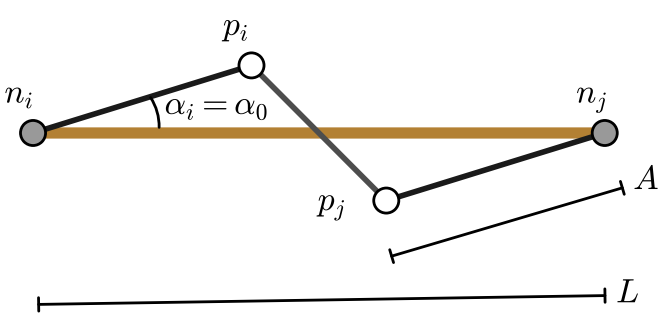

(b)

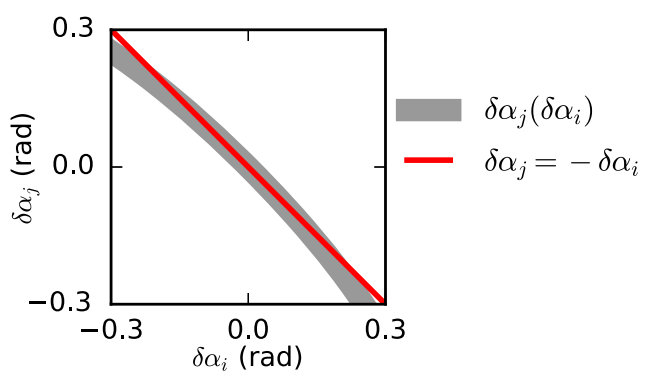

(c)

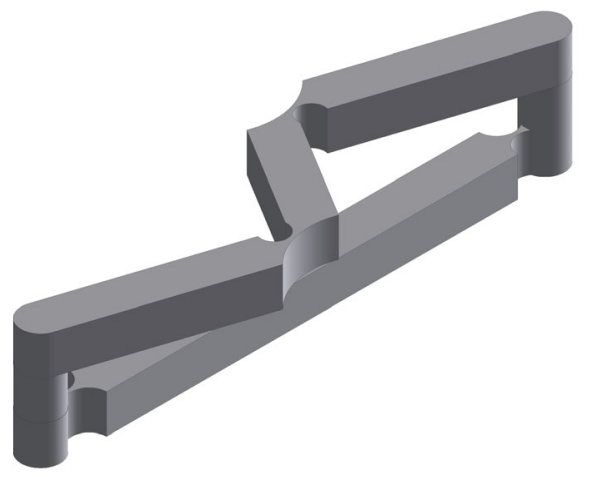

FIG. 8. An alternative to the geared link. (a) The link constrains the motions of freely pivoting nodes $n_{i}, n_{j}$ (gray disks); it is symmetric under in-plane rotations by $\pi \mathrm{rad}$. The nodes are connected by a rigid beam of length $L$ (orange line). Node $n_{i}$ is connected to subnode $p_{i}$ (white disk) by a rigid subbeam (black line) of length $A$ at an angle $\alpha_{0}$. Subnodes $p_{i}, p_{j}$ are interconnected by a rigid subbeam of length $l$ (gray line). The beam of length $L$ constrains the distance between nodes $n_{i}, n_{j}$, and the subbeam mechanism ensures that the nodes counterrotate in the linear regime. (b) Numerical calculation of the relationship between deviations $\delta \alpha_{i}, \delta \alpha_{j}$ from the angle $\alpha_{0}$ at nodes $n_{i}, n_{j}$ (gray line), for the link design shown in (a). Here, $\alpha_{0}=0.3 \mathrm{rad}$ and $A / L=0.4$. In the linear regime, the relationship shows excellent agreement with $\delta \alpha_{j}=-\delta \alpha_{i}$ (red line), which describes pure counterrotation of the nodes. (c) A possible continuum 3D design for the link. Thin hinges accommodate low-energy pivoting at nodal points within the structure.

$$
\begin{aligned}
& \vec{p}_{1}=A \cos \left(\alpha_{i}\right) \hat{x}+A \sin \left(\alpha_{i}\right) \hat{y}, \\
& \vec{p}_{2}=\left[L-A \cos \left(\alpha_{j}\right)\right] \hat{x}-A \sin \left(\alpha_{j}\right) \hat{y} ;
\end{aligned}
$$

setting (1) $\alpha_{i}=\alpha_{j}=\alpha_{0}$ and (2) $\alpha_{i}=\alpha_{0}+\delta \alpha_{i}$ and $\alpha_{j}=\alpha_{0}+\delta \alpha_{j}$, we obtain a system of equations that we can solve in a straightforward manner for $\delta \alpha_{i}$ and $\delta \alpha_{j}$ to linear order. This calculation results in the relation of 
Eq. (F1), showing that the nodes in this mechanism purely counterrotate in the linear regime. From numerical calculations, deviations from this counterrotating behavior are smaller than $5 \%$ up to $\delta \alpha_{i} \sim 0.2 \alpha_{0}$, at least where $0.25<$ $A / L<0.65$ and $0.15<\alpha_{0}<0.55$ rad [Fig. 8(b)].

Note that for angle deviations $\delta \alpha$ outside of the linear regime, the linear relation between nodal angles is no longer valid. In addition, if the nodal angles are such that $\vec{n}_{i}, \vec{p}_{1}$ and $\vec{p}_{2}$ are collinear, the mechanism may convert to one in which corotations (rather than counterrotations) of the nodes are enforced. In addition, the desired behavior breaks down if the $L$ beam is allowed to stretch significantly (similarly to the geared link). Deviations $\delta L$ in the length $L$ are coupled to corotations of the nodes, or shearing of the mechanism:

$$
\delta \alpha_{i}=\delta \alpha_{j}=\frac{2 A-L}{2 A L \alpha_{0}} \delta L
$$

A possible continuum realization of the link design is shown in Fig. 8(c). This design may be used to build up a lattice with many unit cells that forms a continuum solid, which can be manufactured via 3D printing or other additive manufacturing techniques. In a network built up out of such elements, all modes will cost a finite amount of energy due to bending at thin hinges inside the link. States of self-stress will exhibit themselves as stretching, buckling, or bending of these thin hinges rather than failure of the thicker beams. Self-intersections can be avoided with layering and appropriate choices of $A / L$ and $\alpha$.

[1] D. J. de Solla Price, On the Origin of Clockwork, Perpetual Motion Devices, and the Compass (United States National Museum, Washington, DC, 1959).

[2] A. E. Musson and E. Robinson, Science and Technology in the Industrial Revolution (Manchester University Press, Manchester, 1969).

[3] R. Di Leonardo, L. Angelani, D. Dell'Arciprete, G. Ruocco, V. Iebba, S. Schippa, M. P. Conte, F. Mecarini, F. De Angelis, and E. Di Fabrizio, Bacterial Ratchet Motors, Proc. Natl. Acad. Sci. U.S.A. 107, 9541 (2010).

[4] T. Mirkovic, N. S. Zacharia, G. D. Scholes, and G. A. Ozin, Nanolocomotion-Catalytic Nanomotors and Nanorotors, Small 6, 159 (2010).

[5] C. Maggi, F. Saglimbeni, M. Dipalo, F. De Angelis, and R. Di Leonardo, Micromotors with Asymmetric Shape that Efficiently Convert Light into Work by Thermocapillary Effect, Nat. Commun. 6, 7855 (2015).

[6] I. Williams, E. C. Oguz, T. Speck, P. Bartlett, H. Löwen, and C. Patrick Royall, Transmission of Torque at the Nanoscale, Nat. Phys. 12, 98 (2015).

[7] S. Erbas-Cakmak, D. A. Leigh, C. T. McTernan, and A. L. Nussbaumer, Artificial Molecular Machines, Chem. Rev. 115, 10081 (2015).
[8] M. Kadic, T. Bückmann, R. Schittny, and M. Wegener, Metamaterials beyond Electromagnetism, Rep. Prog. Phys. 76, 126501 (2013).

[9] J. Christensen, M. Kadic, O. Kraft, and M. Wegener, Vibrant Times for Mechanical Metamaterials, MRS Commun. 5, 453 (2015).

[10] R. Lakes, Foam Structures with a Negative Poisson's Ratio, Science 235, 1038 (1987).

[11] J. N. Grima, A. Alderson, and K. E. Evans, Auxetic Behaviour from Rotating Rigid Units, Phys. Status Solidi B 242, 561 (2005).

[12] G. W. Milton and A. V. Cherkaev, Which Elasticity Tensors are Realizable?, J. Eng. Mater. Technol. 117, 483 (1995).

[13] M. Kadic, T. Bückmann, N. Stenger, M. Thiel, and M. Wegener, On the Practicability of Pentamode Mechanical Metamaterials, Appl. Phys. Lett. 100, 191901 (2012).

[14] S. Shan, S. H. Kang, P. Wang, C. Qu, S. Shian, E. R. Chen, and K. Bertoldi, Harnessing Multiple Folding Mechanisms in Soft Periodic Structures for Tunable Control of Elastic Waves, Adv. Funct. Mater. 24, 4935 (2014).

[15] P. Seppecher, J.-J. Alibert, and F. D. Isola, Linear Elastic Trusses Leading to Continua with Exotic Mechanical Interactions, J. Phys. Conf. Ser. 319, 012018 (2011).

[16] C. L. Kane and T. C. Lubensky, Topological Boundary Modes in Isostatic Lattices, Nat. Phys. 10, 39 (2014).

[17] D. Z. Rocklin, Bryan Gin-ge Chen, M. Falk, V. Vitelli, and T. C. Lubensky, Mechanical Weyl Modes in Topological Maxwell Lattices, Phys. Rev. Lett. 116, 135503 (2016).

[18] H. C. Po, Y. Bahri, and A. Vishwanath, Phonon Analog of Topological Nodal Semimetals, Phys. Rev. B 93, 205158 (2016).

[19] M.Z. Hasan and C.L. Kane, Colloquium: Topological Insulators, Rev. Mod. Phys. 82, 3045 (2010).

[20] X.-L. Qi and S.-C. Zhang, Topological Insulators and Superconductors, Rev. Mod. Phys. 83, 1057 (2011).

[21] L. Lu, J. D. Joannopoulos, and M. Soljačić, Topological Photonics, Nat. Photonics 8, 821 (2014).

[22] E. Prodan and C. Prodan, Topological Phonon Modes and Their Role in Dynamic Instability of Microtubules, Phys. Rev. Lett. 103, 248101 (2009).

[23] R. Susstrunk and S. D. Huber, Observation of Phononic Helical Edge States in a Mechanical Topological Insulator, Science 349, 47 (2015).

[24] Y.-T. Wang, P.-G. Luan, and S. Zhang, Coriolis Force Induced Topological Order for Classical Mechanical Vibrations, New J. Phys. 17, 073031 (2015).

[25] P. Wang, L. Lu, and K. Bertoldi, Topological Phononic Crystals with One-Way Elastic Edge Waves, Phys. Rev. Lett. 115, 104302 (2015).

[26] S. Hossein Mousavi, A. B. Khanikaev, and Z. Wang, Topologically Protected Elastic Waves in Phononic Metamaterials, Nat. Commun. 6, 8682 (2015).

[27] A. B. Khanikaev, R. Fleury, S. Hossein Mousavi, and A. Alù, Topologically Robust Sound Propagation in an Angular-Momentum-Biased Graphene-like Resonator Lattice, Nat. Commun. 6, 8260 (2015).

[28] L. M. Nash, D. Kleckner, A. Read, V. Vitelli, A. M. Turner, and W. T. M. Irvine, Topological Mechanics of Gyroscopic Metamaterials, Proc. Natl. Acad. Sci. U.S.A. 112, 14495 (2015). 
[29] S. D. Huber, Topological Mechanics, Nat. Phys. 12, 621 (2016).

[30] B. Gin-ge Chen, N. Upadhyaya, and V. Vitelli, Nonlinear Conduction via Solitons in a Topological Mechanical Insulator, Proc. Natl. Acad. Sci. U.S.A. 111, 13004 (2014).

[31] J. Paulose, B. Gin-ge Chen, and V. Vitelli, Topological Modes Bound to Dislocations in Mechanical Metamaterials, Nat. Phys. 11, 153 (2015).

[32] J. Paulose, A.S. Meeussen, and V. Vitelli, Selective Buckling via States of Self-Stress in Topological Metamaterials, Proc. Natl. Acad. Sci. U.S.A. 112, 7639 (2015).

[33] Bryan Gin-ge Chen, B. Liu, A. A. Evans, J. Paulose, I. Cohen, V. Vitelli, and C. D. Santangelo, Topological Mechanics of Origami and Kirigami, Phys. Rev. Lett. 116, 135501 (2016).

[34] D. Zeb Rocklin, S. Zhou, K. Sun, and X. Mao, Transformable Topological Mechanical Metamaterials, arXiv: 1510.06389.

[35] S. Guest and J. Hutchinson, On the Determinacy of Repetitive Structures, J. Mech. Phys. Solids 51, 383 (2003).

[36] V. Kapko, M. M. J. Treacy, M. F. Thorpe, and S. D. Guest, On the Collapse of Locally Isostatic Networks, Proc. R. Soc. A 465, 3517 (2009).

[37] T. C. Lubensky, C. L. Kane, X. Mao, A. Souslov, and K. Sun, Phonons and Elasticity in Critically Coordinated Lattices, Rep. Prog. Phys. 78, 073901 (2015).

[38] C. R. Calladine, Buckminster Fuller's Tensegrity Structures and Clerk Maxwell's Rules for the Construction of Stiff Frames, Int. J. Solids Struct. 14, 161 (1978).

[39] J. C. Maxwell, On the Calculation of the Equilibrium and Stiffness of Frames, Philos. Mag. 27, 294 (1864).

[40] K. Shundyak, M. van Hecke, and W. van Saarloos, Force Mobilization and Generalized Isostaticity in Jammed Packings of Frictional Grains, Phys. Rev. E 75, 010301 (2007).

[41] S. Papanikolaou, C. S. O'Hern, and M. D. Shattuck, Isostaticity at Frictional Jamming, Phys. Rev. Lett. 110, 198002 (2013).
[42] S. Henkes, D. A. Quint, Y. Fily, and J. M. Schwarz, Rigid Cluster Decomposition Reveals Criticality in Frictional Jamming, Phys. Rev. Lett. 116, 028301 (2016).

[43] A. J. Liu and S. R. Nagel, The Jamming Transition and the Marginally Jammed Solid, Annu. Rev. Condens. Matter Phys. 1, 347 (2010).

[44] D. M. Sussman, O. Stenull, and T. C. Lubensky, Topological Boundary Modes in Jammed Matter, Soft Matter 12, 6079 (2016).

[45] C. R. Scullard, Exact Site Percolation Thresholds Using a Site-to-Bond Transformation and the Star-Triangle Transformation, Phys. Rev. E 73, 016107 (2006).

[46] K. Sun, A. Souslov, X. Mao, and T. C. Lubensky, Surface Phonons, Elastic Response, and Conformal Invariance in Twisted Kagome Lattices, Proc. Natl. Acad. Sci. U.S.A. 109, 12369 (2012).

[47] See Supplemental Material at http://link.aps.org/ supplemental/10.1103/PhysRevX.6.041029 for movies illustrating topological and mechanical properties of the gear networks under study.

[48] D. Prall and R. S. Lakes, Properties of a Chiral Honeycomb with a Poisson's Ratio of -1 , Int. J. Mech. Sci. 39, 305 (1997).

[49] J. B. Conway, Mathematical Connections: A Capstone Course (American Mathematical Society, Providence, 2010).

[50] G. Gordon, Workable Gears, Archimedian Solids and Planar Bipartite Graphs, Am. Math. Mon. 101, 527 (1994).

[51] J. Calì, D. A. Calian, C. Amati, R. Kleinberger, A. Steed, J. Kautz, and T. Weyrich, 3D-Printing of Non-Assembly, Articulated Models, ACM Trans. Graph. 31, 130 (2012).

[52] R. MacCurdy, R. Katzschmann, Y. Kim, and D. Rus, "Printable Hydraulics: A Method for Fabricating Robots by $3 D$ Co-Printing Solids and Liquids," in Proceedings of the IEEE International Conference on Robotics and Automation (IEEE, New York, 2016).

[53] R. G. Hutchinson and N. A. Fleck, Microarchitectured Cellular Solids-The Hunt for Statically Determinate Periodic Trusses, Z. Angew. Math. Mech. 85, 607 (2005). 